\title{
The Black Box Module for Low-Level Fluorescence Detection Targeting Optogenetic Studies
}

\author{
Elizabeth Maret ${ }^{\mathrm{a}}$ and Mohamed Diagne ${ }^{\mathrm{a}}$
}

\begin{abstract}
In optogenetics, genetic labeling by enhanced yellow fluorescent protein (EYFP) indicates transfected neurons expressing lightgated channel proteins, Channelrhodopsin-2 (ChR2) and Halorhodopsin (NpHR) for millisecond timescale optical manipulation of neurons. However, poor spatial understanding of transfection relative to probing is responsible for blind stimulation in complex mammalian systems and necessitates histological analysis of brain tissue. Repeatable optogenetic studies on welltrained and valuable subjects are therefore impossible. We report here the Black Box Module as a novel fluorescence detection system targeting EYFP emission and used in conjunction with a co-axial waveguide "optrode." Results of the Black Box Module's fluorescence detection performance in a bench-top environment simulating EYFP fluorescence with rhodamine $6 \mathrm{~g}$ are presented. We show the Box's filtration system to be sensitive to the EYFP-like fluorescence of rhodamine and its concentration dependent emissions behaviors. We anticipate these results to be the initial foundation in integrating the Box into optogenetic studies as a tool for locating areas of ChR2 and NpHR transfection.
\end{abstract}

Keywords: Enhanced Yellow Fluorescent Protein, Black Box Module, Rhodamine 6G, fluorescence

\subsection{Introduction}

Optogenetics enables cell-type specific targeting and millisecond timescale control of neuron action potential events through viral vector transfection of light-responsive channel proteins. Light-gated channel proteins Channelrhodopsin-2 (ChR2) and Halorhodopsin (NpHR) are conjoined with enhanced yellow fluorescent proteins (EYFPs) to visually label areas of neuron transfection ${ }^{[1,2,4,6,8]}$. Dualfunction optical stimulation and electrical recording of neuron action potential events are accomplished through an "optrode," a waveguide-based neuroprobe ${ }^{[10]}$.

While the optrode has shown its value as a tool in precise stimulation and collection of electrical action potentials when located in an optogenetically transfected areas, locating areas of transfection remains a challenge ${ }^{[4]}$. This leads to blind probing, preventing repeatable studies and the possibility of studies focused on brain layer targeting ${ }^{[4,11]}$. Collection of fluorescence marking $\mathrm{ChR} 2$ and NpHR expressing cells has initiated a new generation of low-light optical detection for systems such as one-photon and two-photon fluorescence microendoscopy and in vivo imaging at the single neuron level ${ }^{[3]}$. Despite these advances, spatial understanding of optical probing relative to areas of transfection is limited. This compromises experimental success and requires histological analysis of subjects to determine transfection patterns ${ }^{[4]}$. It is thus both desirable and economic to perform multiple optogenetic studies on valuable and well-trained test subjects.

To address this issue, we present the Black Box Module used in tandem with the optrode, as a novel system to collect fluorescence from optogenetically modified neurons exhibiting EYFP expression as is used to label ChR2 and $\mathrm{NpHR}$ in optogenetics. Using the optrode's waveguide fiber core, in addition to its simultaneous optical stimulation and electrical recording dual-functionality, we believe the optrode when used with the Black Box Module can also be used for optical fluorescence collection. Hence, the optrode-Box combination can be used to indicate locations of transfection.
We substantiate this prediction by demonstrating how light collected by the optrode and delivered through its optical fiber into the Box isolates for EYFP fluorescence and in addition is sensitive to concentration dependent properties of rhodamine $6 \mathrm{~g}$. This is achieved through a series of filters within the light-tight environment of the Black Box. Highly filtered fluoresced light is detected by photomultiplier tube (PMT). Thus a signal detected by the PMT can verify the optrode's position in an optogenetically modified environment while no signal indicates and non-optogenetic environment.

Our Black Box system uses free space coupling in a light-tight environment, opposed to the split fiber fluorescence detection system of Diester et al $(2011)^{[4]}$. We believe this to be an improvement over the shared core of the split waveguide fiber because it allows for increased opportunities of optical filtration to isolate for fluorescent light. We present the results of a study using the Black Box to detect concentration dependent emissions behavior in a fabricated bench-top optogenetic environment using rhodamine $6 \mathrm{~g}$ fluorescent dye, which mimics EYFP stimulation and emissions. The results show the optrode-Box set up to be sensitive to rhodamine $6 \mathrm{~g}$ 's concentration dependent emissions wavelength shift behavior while maintaining the integrity of its filtration system.

\subsection{Experimental Procedure}

EYFPs have a quantum yield of $0.61^{[9]}$. That means that in optogenetic environments for every EYFP emission event there are $64 \%$ more laser photons than fluorescence photons. Hence, key to transfection labeling fluorescence detection is creating an environment that can isolate detection for fluorescence. The Black Box Module (Figure 1) is an 18 in. $x$ 12 in. x 7.5 in. light-tight enclosure made from $3 / 8$ in. thick black anodized aluminum plates, containing an optical filtration system isolating for EYFP emission (525 nm peak) (Figure 2). The stimulating laser (Opto Engine), emitting at $473 \mathrm{~nm}$, is fixed within the box and free-space couples to a 
dichroic beamsplitter (Semrock Brightline FF520-Di02). Initially, laser light is directed perpendicular from its source by the beamsplitter and free-space coupled to an optical fiber, which passes through the wall of the Box to butt-couple with the optrode. Laser light then exits the optrode to illuminate a rhodamine solution and stimulate fluorescence. Fluorescence, scattered laser light, and ambient light are collected by the optrode's waveguide core to re-enter the Box. Once inside, collected light exits the fiber to strike the dichroic mirror at normal incidence, allowing light above the $520 \mathrm{~nm}$ edge wavelength to pass through. The collected light then passes through two identical optical filters (Semrock Brightline FF01-542/27) with transmission bandwidth 528.5-555.5 nm (Figure 2). A pin-hole spatial filter controls beam divergence before incidence on the photomultiplier tube (PMT) (PerkinElmer MP 942). The PMT is positioned within the wall of the Box and an oscilloscope connects to the externally protruding end.

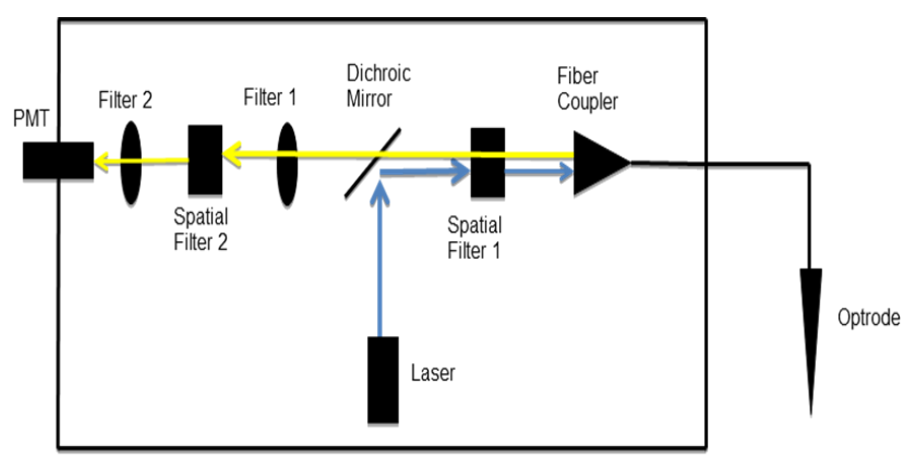

Figure 1: Black Box Module stimulation and collection schematic.

The Box's method of EYFP fluorescence isolation using optical filters is highlighted in Figure 2, showing the filter's transmission bandwidth to isolate for EYFP's peak emissions at $525 \mathrm{~nm}$ and blocking out laser light at $473 \mathrm{~nm}$. As previously stated, initial fluorescence detection as measured by the Box was performed using rhodamine $6 \mathrm{~g}$ fluorescent dye in an ethanol solution to mimic EYFP emissions. Rhodamine's stimulation and emissions spectra mimic that of EYFP's as shown by the close overlap of spectra in Figure 3.

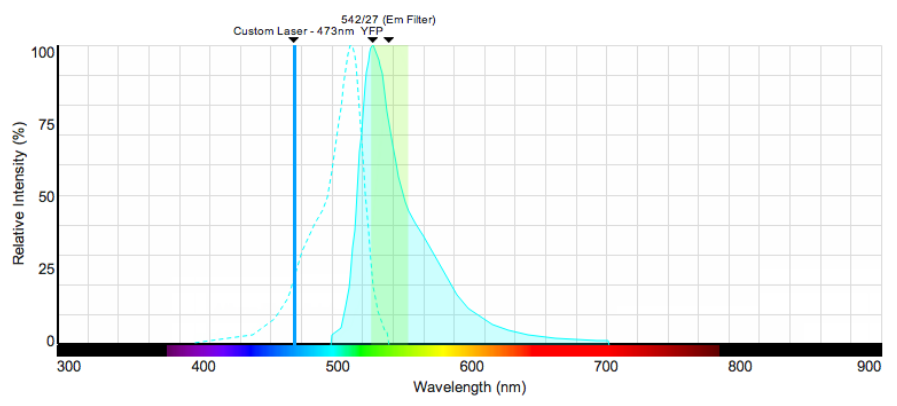

Figure 2: EYFP absorption and emissions spectrum (blue). The light green shading highlights the optical filter bandwidth (542/27 (EM Filter)). The solid blue line indicates the $473 \mathrm{~nm}$ stimulating laser light (courtesy of Invitrogen Fluorescence SpectraViewer).

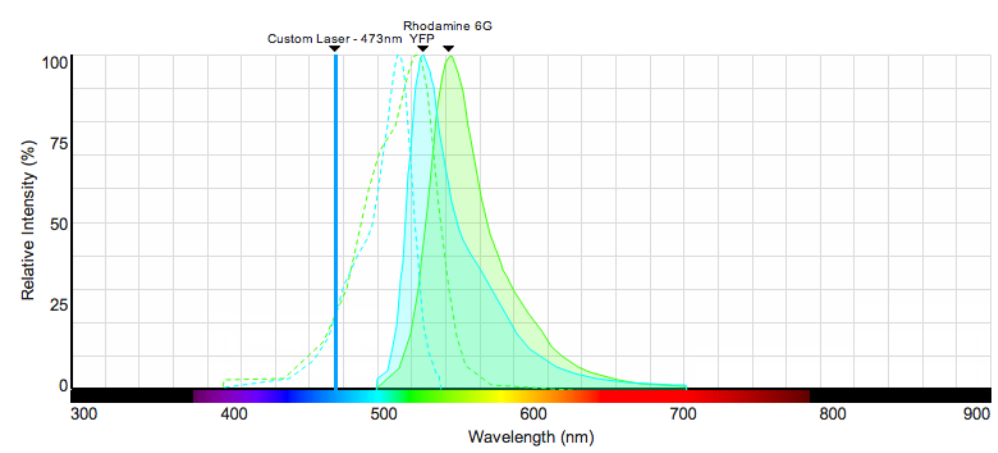

Figure 3: EYFP (blue) and rhodamine 6g (green) absorption and emissions spectra. The stimulating laser light is represented as the solid line at $473 \mathrm{~nm}$. (Courtesy of Invitrogen Fluorescence SpectraViewer)

Literature reports rhodamine to exhibit concentration dependent emissions qualities. We used the Black Box to measure fluorescence over a range of concentrations in attempt to observe the Box's sensitivity to the concentration dependent behavior. Fluorescence response was measured as photon count events over $1 \times 10^{-7} \mathrm{M}-1 \times 10^{-2} \mathrm{M}$ rhodamine concentrations in an ethanol solution by increments of $1 \times 10^{-7}$ $\mathrm{M}, 5 \times 10^{-7} \mathrm{M}, 1 \times 10^{-6} \mathrm{M}, 5 \times 10^{-6} \mathrm{M}, 1 \times 10^{-5} \mathrm{M}$, and so on until $1 \times 10^{-2} \mathrm{M}$. Thus, fluorescence photon counts were taken at a total of 11 concentrations. Fluorescence response was measured as the frequency of photon count events per second as recorded by the PMT. A base noise count of photon events on the PMT was measured in a $100 \%$ ethanol solution between each fluorescence measurement. Base noise counts were directly related to the stimulating laser intensity. Measurements for the 11 concentrations were taken at four base count intensities: $1 \mathrm{kHz}, 10 \mathrm{kHz}, 100 \mathrm{kHz}$, and $1 \mathrm{MHz}$. The relative fluorescence response $\mathrm{N}$ relative to the base count was found by the equation:

$$
N=\frac{c_{m}-c_{b}}{c_{b}}
$$

where $c_{m}$ is the measured fluorescence count and $c_{b}$ is the base count.

Rhodamine exhibits secondary emissions from the approximately $475-525 \mathrm{~nm}$ overlap in its absorption and emissions spectrum ${ }^{[5]}$. Measuring emissions at wavelengths outside the area of overlap show low concentrations of rhodamine to have a constant linear quantum yield in the $1 \times 10^{-6}$ $\mathrm{M}-1.5 \times 10^{-4} \mathrm{M}$ range ${ }^{[5]}$. Optical filters in the Box isolate for fluorescence outside the overlap in the 528.5-555.5 nm transmission bandwidth of the filter. Thus, the Box's measured fluorescence response is expected to also exhibit a constant linear trend until $1.5 \times 10^{-4} \mathrm{M}$.

As rhodamine concentrations increase to $2 \times 10^{-4} \mathrm{M}$, the monomer solution forms polymers. The solution's change in composition alters the light interaction with the molecule, and rhodamine's emissions spectrum shifts towards infrared wavelengths as concentration increases ${ }^{[5,7]}$. Kubin and Fletcher (1982) ${ }^{[7]}$ report that at $1.5 \times 10^{-7} \mathrm{M}$, peak emission occurs at approximately $525 \mathrm{~nm}$ compared to approximately $580 \mathrm{~nm}$ emissions at $1 \times 10^{-3} \mathrm{M}^{[7]}$. It must be noted that the Box's filters block out wavelengths above $555.5 \mathrm{~nm}$ and Kubin and Fletcher 
cite no upper limit to the concentration dependent red shift behavior. Therefore, as rhodamine concentrations increase and the fluorescence emissions redshifts, the Box's measured response rate is expected to slow and show a decrease in response as it reaches concentrations exceeding $1 \times 10^{-3} \mathrm{M}$.

Based on the properties of rhodamine described above there are two key properties the Box's sensitivity must accommodate to be considered for optogenetic environments. First, it must pick up on the low concentration linear quantum yield to verify its sensitivity to fluorescence detection. Second, due to rhodamine's red-shift under increasing concentrations, the Box's measurements must show a decline in count rates at concentrations beyond $2 \times 10^{-4} \mathrm{M}$ to claim the validity of the optical filters.

\subsection{Results}

\subsection{Rhodamine Redshift}

Data collectedover the $1 \mathrm{kHz}, 10 \mathrm{kHz}, 100 \mathrm{kHz}$ and $1 \mathrm{MHz}$ base counts are shown in Figure 4. Responses across the $1 \mathrm{kHz}, 10 \mathrm{kHz}$ and $100 \mathrm{kHz}$ base counts show the relative response rate to notably slow between $1 \times 10^{-3} \mathrm{M}$ and $5 \times 10^{-3}$

M. Responses for the $1 \mathrm{MHz}$ base count saturated the sensitivity parameters of the PMT at concentrations above $1 \times 10^{-5} \mathrm{M}$ and hence Figure 4 shows no fluorescence response for concentrations above $1 \times 10^{-5} \mathrm{M}$.

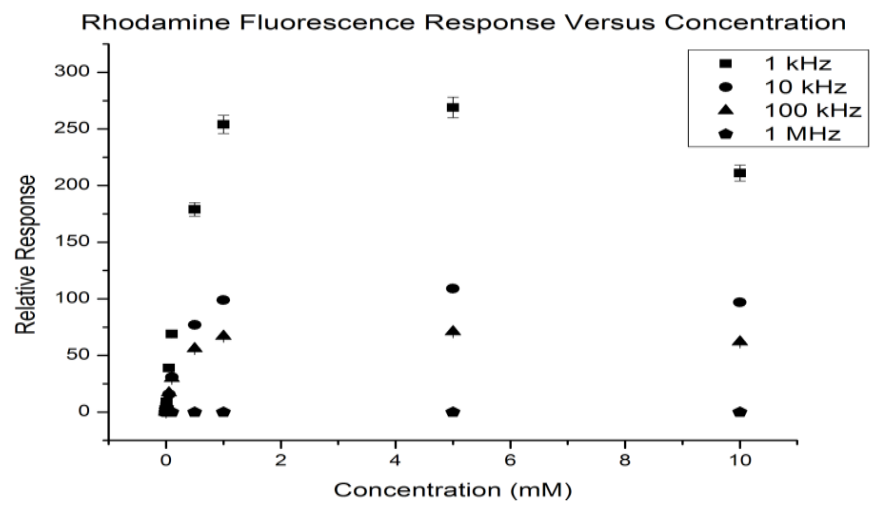

Figure 4: Rhodamine's relative fluorescence response for 1 $\mathrm{kHz}, 10 \mathrm{kHz}, 100 \mathrm{kHz}$, and $1 \mathrm{MHz}$ base counts of $1 \times 10^{-7}-10^{-}$ ${ }^{3} \mathrm{M}$ concentrations.

\subsection{Low-Concentration Constant Quantum Yield}

Examining the $1 \mathrm{kHz}$ base count behavior, Figure 5 shows the constant quantum yield behavior as measured by the Box for the $1 \times 10^{-7}-1 \times 10^{-4} \mathrm{M}$ concentration range. This behavior is similarly observed at the $10 \mathrm{kHz}, 100 \mathrm{kHz}$ and 1 $\mathrm{MHz}$ base counts (not presented here).

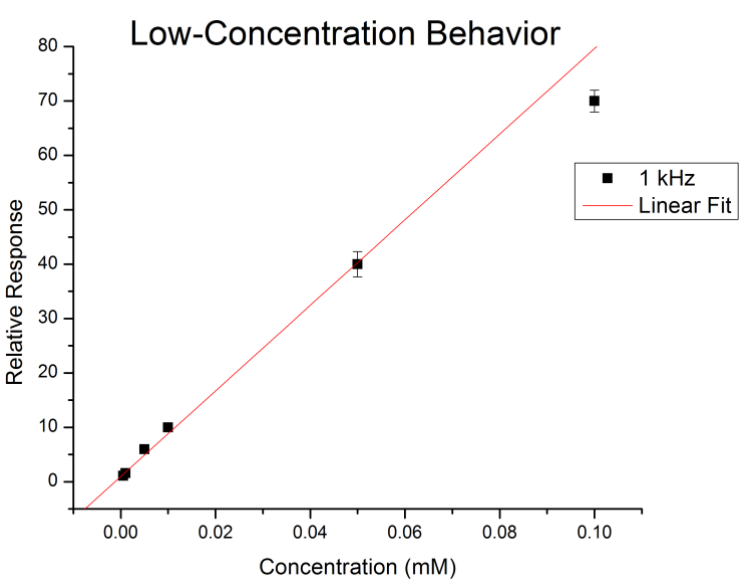

Figure 5: Rhodamine's low-concentration constant quantum yield for $1 \mathrm{kHz}$ base count.

\subsection{Discussion}

As expressed in Section 3.1, Figure 4 presents rhodamine's relative response across all base counts. Within the $1 \times 10^{-3} \mathrm{M}$ and $5 \times 10^{-3} \mathrm{M}$ concentration range, the $1 \mathrm{kHz}, 10$ $\mathrm{kHz}$ and $100 \mathrm{kHz}$ base counts show the response rate to slow. This range corresponds to the region in which Kubin and Fletcher (1982) ${ }^{[7]}$ report a value of approximately $580 \mathrm{~nm}$ for rhodamine peak emissions, which lies outside the transmission bandwidth of the Box's filters. Moreover, Figure 4 shows that within this range the fluorescent response rate decreases between $5 \times 10^{-3} \mathrm{M}$ and $1 \times 10^{-2} \mathrm{M}$. Since Kubin and Fletcher do not cite an upper limit in rhodamine's red shift behavior, we believe the red shift behavior continues such that the peak emissions wavelength shifts further outside the filters' transmission bandwidth. Thus, as concentration increases beyond $1 \times 10^{-3} \mathrm{M}$, more fluorescence is filtered out, causing a decrease in detection by the PMT.

Section 3.2 described the Box's detection of rhodamine's low-concentration behavior. The linear trend of the data confirms rhodamine's expected constant quantum yield at concentrations below $2 \times 10^{-4} \mathrm{M}$, as expressed by Fischer and Georges (1996) ${ }^{[5]}$.

The Box's sensitivity to the concentration dependent redshift and constant low-concentration linear behavior of rhodamine confirms its ability to isolate for EYFP-like fluorescence. This presents the prospect of addressing the explicit concern of Diester et al (2011) ${ }^{[4]}$ in the need for improved spatial understanding of optogenetic probing to locate areas of transfection. Considering that EYFP peak emissions is understood to be constant compared to rhodamine's concentration emissions, we expect the Box's filtration and detection system to be able to separate and detect EYFP fluorescence from scattering laser and noise light signals. To verify this, the next step is to use the Black Box to measure actual EYFP fluorescence. Provided this next study is successful, we predict the Box-optrode system can eventually be used to locate areas of Chr2- or NpHR-EYFP expressing neurons indicated by collecting positive fluorescence signals. 
Before the Box-optrode system is regularly integrated into optogenetic studies it is necessary to calibrate the system for areas of transfection. The potency of viral vector transfection and how it spreads through a volume is still under examination ${ }^{[4]}$. Thus it is important to examine the event count signatures associated with differing EYFP potencies as a further means of achieving spatial understanding when probing.

\subsection{Conclusion}

By the ability of the Box's low-level fluorescence detection system used with the optrode to detect expected behaviors in rhodamine's concentration dependent fluorescence response, we are confident in its function as a tool for fluorescence detection. This study also displays the optrode-Box combination has the potential to jointly achieve optical stimulation, optical and electrical recording. We anticipate the Box's integration into in vivo optogenetic studies to improve spatial understanding in optogenetic probing and significantly reduce the need for terminal histological analysis on expensive test subjects.

\section{Acknowledgements}

This work was supported by the Defense Advanced Research Projects Agency (Repair Program) subcontract no. 2548004047135-B, and a startup fund from the Dean of Faculty Office at Connecticut College. The authors would like to extend their sincere gratitude and appreciation to Professor Arto Nurmikko of Brown University and his team for this incredible undergraduate research opportunity. Thanks to Ilker Ozden and Jing Wang from Brown University for their contribution in optimizing the module performance.

\section{Role of the Funding Source}

This work was supported by the Defense Advanced Research Projects Agency (Repair Program) subcontract no. 25480040$47135-\mathrm{B}$, and funds from the Dean of Faculty Office at Connecticut College.

\section{References:}

1. Bamann, C., Nagel, G., Bamberg, E. 2010. Microbial rhodopsins in the spotlight. Curr. Opin. Neurobiol. 20: 610-616.

2. Boyden, E.S., Zhang, F., Bamberg, E., Nagel G., and Deisseroth, K. 2005. Millisecond-timescale, genetically targeted optical control of neural activity. Nat Neurosci. 8:1263-1268.

3. Deisseroth, K., Feng, G., Majewska, A.K., Miesenbock, G., Schnitzer, M.J. 2006. Next-generation optical technologies for illuminating genetically targeted brain circuits. J. Neurosci. 26:10380-10386.

4. Diester, I., Kaufman, M.T., Mogri, M., Pashaie, R., Goo, W., Yizhar, O., Ramakrishnan, C., Deisseroth, K., and Shenoy, K.V. 2011. An optogenetic toolbox designed for primates. Nat Neurosci. 14:387-397.

5. Fischer, M., Georges, J. 1996. Fluorescence quantum yield of rhodamine $6 \mathrm{G}$ in ethanol as a function of concentration using thermal lens spectrometry. Chem. Phys. Lett. 260:115-118.

6. Gradinaru, V., Zhang, F., Ramakrishnan, C., Mattis, J., Prakash, R., Diester, I., Goshen, I., Thompson, K.R., and Deisseroth, K. 2010. Molecular and cellular approaches for diversifying and extending optogenetics. Cell. 141:154-165.

7. Kubin, R.F., Flectcher, A.N. 1982. Fluorescence Quantum Yields of Some Rhodamine Dyes. J. Lumin. 27:455462.

8. Nagel, G., Szellas, T., Huhn, W., Kateriya, S., Adeishvili, N., Berthold, P., Ollig, D., Hegemann, P., and Bamberg, E. 2003. Channelrhodopsin-2, a directly light-gated cation-selective membrane channel. Proc. Natl. Acad. Sci. U.S.A. 100:13940-13945.

9. Shaner, N.C., Steinbach, P.A., and Tsien, R.Y. 2005. A guide to choosing fluorescent proteins. Nat Methods. 2:905-909.

10. Wang, J., Borton, D.A., Zhang, J., Burwell, R.D., and Nurmikko, A.V. 2010. A neurophotonic device for stimulation and recording of neural microcircuits. $32^{\text {nd }}$ Annual International Conference of the IEEE EMBS. 2935-2938.

11. Yizhar, O., Fenno, L. E., Davidson, T. J., Mogri, M, Deisseroth, K. 2011. Optogenetics in neural systems. Neuron. 71:9-34. 